\title{
Steady State Characteristics and Methods of Calculations of Stady State of Synchronous Motors
}

\author{
Aleksey N. Filatov, \\ Evgenia Yu. Sizganova*, Vasiliy I. Panteleev, \\ Roman A. Petukhov and Gennadiy A. Pilyugin \\ Siberian Federal University \\ 79 Svobodny, Krasnoyarsk, 660041, Russia
}

Received 08.04.2015, received in revised form 19.07.2015, accepted 20.08.2015

Analitical dependensies describing relations between an active power, reactive power stator voltage, stator current are obtained. They are take into account salient pole of motor and variation of satura-tion of magnetis circuit and allow to calculate stady state of synchronous motor at minimum of basic data.

Keywords: synchronous electric motor, active power, reactive power, satiation of magnetic chain.

DOI: $10.17516 / 1999-494 X-2015-8-6-795-801$.

\section{Статические характеристики \\ и методы расчета установившихся режимов работы синхронных двигателей}

А.Н. Филатов, Е.Ю. Сизганова, В.И. Пантелеев, Р.А. Петухов, Г.А. Пилюгин Сибирский федеральный университет Россия, 660041, Красноярск, пр. Свободный, 79

\footnotetext{
Приводятся аналитические зависимости, описывающие связи между активной мощностью, реактивной мощностью, напряжением статора, током статора и током возбуждения. Они учитывают явнополюсность ротора и изменение насыщения магнитной цепи и позволяют при минимальном объеме исходных данных рассчитать установившийся режим работь синхронного двигателя.
}

Ключевые слова: синхронный двигатель, активная мощность, реактивная мощность, насыщение магнитной цепи.

(C) Siberian Federal University. All rights reserved

* Corresponding author E-mail address: seu_eset@mail.ru 
При расчетах и оптимизации установившихся режимов систем электроснабжения промышленных предприятий со значительной установленной мощностью синхронных двигателей (СД) необходимы, в числе прочих, аналитические описания связей вида:

$$
\begin{aligned}
& g_{1}\left(P_{1}, Q, U_{1}, I_{1}\right)=0, \\
& g_{2}\left(P_{1}, Q, U_{1}, I_{2}\right)=0, \\
& g_{3}\left(P_{1}, \underline{Q}, U_{1}\right)=0,
\end{aligned}
$$

где $g_{1}, g_{2}, g_{3}$ - неявные функции; $P_{1}, Q$ - активная, кВт и реактивная, кВАр, мощности СД; $U_{1}, I_{1}-$ напряжение, кВ, и ток, А, статора; $I_{2}$ - ток возбуждения, А; $Q, I_{2}$ - нижние предельно допустимые значения реактивной мощности и тока возбуждения из условия осуществимости установившегося режима работы СД.

Статические характеристики СД и методы расчета их установившихся режимов работы (зависимости $Q, I_{1}$ при заданных $P_{1}, U_{1}, I_{2}$ и зависимости $Q, I_{2}$ при заданных $\left.P_{1}, U_{1}\right)$ должны учитывать явнополюсность ротора и изменение насыщения магнитной цепи и использовать минимальное количество исходных данных.

Аналитические описания и методы расчета. Используя результаты работ автора [1], аналитическое описание нормальной характеристики холостого хода СД, предложенное Ю.М. Голодновым [2], и следующие обозначения:

$$
\alpha_{S}=\frac{Q}{S_{\mathrm{H}}}, \quad \beta_{s}=\frac{P_{1}}{S_{\mathrm{H}}}, \quad \underline{u}=\frac{U_{1}}{U_{1 \mathrm{H}}}, \quad \underline{I_{1}}=\frac{I_{1}}{I_{1 \mathrm{H}}}, \quad \underline{I}_{2}=\frac{I_{2}}{I_{2 \mathrm{H}}},
$$

нетрудно показать, что искомые связи (1), (2) могут быть описаны следующими нелинейными уравнениями:

$$
\begin{aligned}
& g_{1}=\alpha_{s}^{2}+\beta_{s}^{2}-\underline{u}^{2} \cdot \underline{I}_{1}^{2}=0, \\
& g_{2}=L\left(\underline{I}_{2}\right)-\psi\left(\alpha_{s}, \beta_{s}, \underline{u}\right)=0, \\
& L\left(\underline{I}_{2}\right)=\varepsilon_{0}^{2}=\varepsilon_{0 \mathrm{H}}^{2} \cdot \underline{I}_{2}^{2}, \\
& \psi\left(\alpha_{s}, \beta_{s}, \underline{u}\right)=\varepsilon_{0}^{2}=\varepsilon^{2}+(\Delta \varepsilon)^{2}+2 \cdot \varepsilon \cdot \Delta \varepsilon \cdot \cos \left(\theta-\delta_{p}\right), \\
& \varepsilon=\frac{1}{\underline{u}} \cdot\left[x_{d} \cdot \beta_{s} \cdot \sin \theta+\left(\underline{u}^{2}+x_{d} \cdot \alpha_{s}\right) \cdot \cos \theta\right], \\
& \Delta \varepsilon=\frac{1,16}{1-0,57 \cdot \varepsilon_{p}} \cdot\left[0,15 \cdot \varepsilon_{p}+\left(0,13 \cdot \varepsilon_{p}-0,0514 \cdot \varepsilon_{p}^{2}\right)^{\frac{1}{2}}\right]-\varepsilon_{p}, \\
& \varepsilon_{p}=\frac{\underline{u^{2}}+x_{p} \cdot \alpha_{s}}{\underline{u} \cdot \cos \delta_{p}}, \\
& \theta=\arctan \frac{x_{q} \cdot \beta_{s}}{\underline{u}^{2}+x_{q} \cdot \alpha_{s}}, \\
& \delta_{p}=\arctan \frac{x_{p} \cdot \beta_{s}}{\underline{u}^{2}+x_{p} \cdot \alpha_{s}},
\end{aligned}
$$


где $S_{\text {н }}$ - номинальное значение полной мощности СД, кВА; $U_{1 \mathrm{H}}, I_{1 \mathrm{H}}-$ номинальные значения напряжения, $\mathrm{KB}$, и тока статора, $\mathrm{A} ; I_{2 н}$ - номинальное значение тока возбуждения, $\mathrm{A} ; \varepsilon_{0}, \varepsilon$ - относительные значения электродвижущей силы (ЭДС) возбуждения с учетом и без учета насыщения магнитной цепи; $\varepsilon_{0 н}-$ номинальное значение ЭДС $\varepsilon_{0} ; \varepsilon_{\mathrm{p}}$ - относительное значение ЭДС Потье; $\Delta \varepsilon$ - относительное значение ЭДС Кимбарка; $\theta$ - угол между ЭДС $\varepsilon$ и напряжением статора, рад.; $\delta_{\mathrm{p}}$ - угол между ЭДС $\varepsilon_{\mathrm{p}}$ и напряжением статора, рад.; $x_{\mathrm{d}}, x_{\mathrm{q}}-$ синхронные индуктивные сопротивления СД по продольной и поперечной осям, отн. ед.; $x_{\mathrm{p}}-$ индуктивное сопротивление Потье, отн. ед.

Задача расчета установившегося режима работы СД на основе полученных математических зависимостей заключается в том, чтобы для заданных относительных значений активной мощности $\beta_{\mathrm{s}}$, напряжения статора $\underline{u}$ и тока возбуждения $\underline{I}_{2}$ найти относительные значения реактивной мощности $\alpha_{\mathrm{s}}$ и тока статора $\underline{I}_{1}$. Для решения задачи необходимы следующие исходные данные о СД: $\cos \varphi_{\mathrm{H}}, x_{\mathrm{d}}, x_{\mathrm{q}}, x_{\mathrm{p}}$, где $\cos \varphi_{\mathrm{H}}-$ номинальное значение коэффициента мощности. Метод решения задачи сводится к следующему:

1) принимают

$$
\alpha_{S}=\sin \varphi_{\mathrm{H}}, \beta_{S}=\cos \varphi_{\mathrm{H}}, \underline{u}=1
$$

и по (12), (13) рассчитывают углы $\delta_{\mathrm{p}}, \theta$, затем по (9), (10), (11) - ЭДС $\varepsilon_{\mathrm{p}}, \Delta \varepsilon$, $\varepsilon$ и по (8) - квадрат номинального значения ЭДС возбуждения $\varepsilon_{0}^{2}=\varepsilon_{0 н}^{2}$;

2) принимают значение $\beta_{\mathrm{s}}, \underline{u}, \underline{I}_{2}$ равными заданным и, решив уравнение (6) с учетом (7) (13), находят искомое значение $\alpha_{\mathrm{s}}$;

3) подставив заданные значения $\beta_{\mathrm{s}}, \underline{u}$ и найденное значение $\alpha_{\mathrm{s}}$ в (5), вычисляют искомое значение $\underline{I}_{1}$.

Корни уравнения (6) находят как абсциссы точек пересечения кривой $\psi\left(\alpha_{\mathrm{s}}, \beta_{\mathrm{s}}, \underline{u}\right)$ с прямой $L\left(\underline{I}_{2}\right)$ (рис. 1a). Точка касания прямой $L\left(\underline{I}_{2}\right)$ с кривой $\psi\left(\alpha_{\mathrm{s}}, \beta_{\mathrm{s}}, \underline{u}\right)$ определит нижнее предельно допустимое - из условия осуществимости установившегося режима - относительное значение реактивной мощности $\alpha_{\mathrm{s}}=\underline{\alpha}_{\mathrm{s}}$ (рис. 1б). Множеству значений $\left\{\alpha_{\mathrm{s}}=\underline{\alpha}_{\mathrm{s}}<\alpha_{\mathrm{s}}<0\right\}$ отвечает режим работы СД с отстающим $\cos \varphi$, значениию $\alpha_{\mathrm{s}}=0$ - режим работы с $\cos \varphi=1,0$ и множеству значений $\left\{\alpha_{\mathrm{s}}: \underline{\alpha}_{\mathrm{s}}>0\right\}$ - режим работы с опережающим $\cos \varphi$.

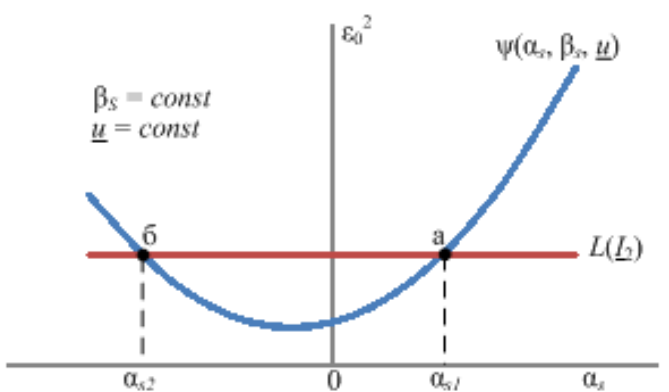

a)

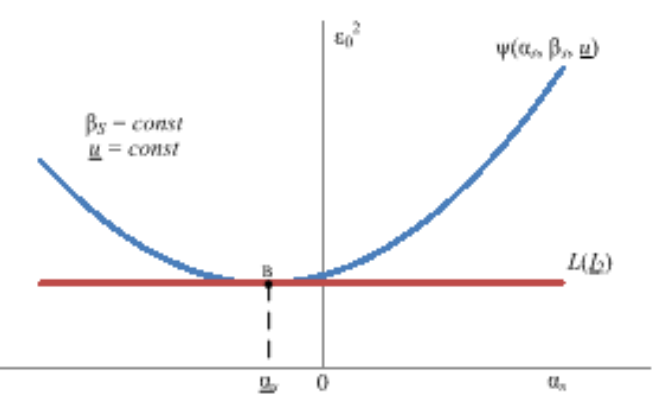

б)

Рис. 1. Определение корней нелинейного уравнения (6) и предельно допустимого относительного значения реактивной мощности по условию осуществимости установившегося режима СД 
Для получения математических зависимостей, описывающих связь (3), достаточно взять производную функцию $\psi\left(\alpha_{\mathrm{s}}, \beta_{\mathrm{s}}, \underline{u}\right)$ по $\alpha_{\mathrm{s}}$ и приравнять полученное выражение нулю:

$$
\begin{aligned}
g_{3}= & \frac{\partial \psi\left(\alpha_{s}, \beta_{s}, \underline{u}\right)}{\partial \alpha_{s}}=\varepsilon \cdot \psi_{1}+\Delta \varepsilon \cdot \psi_{2}+2 \cdot \Delta \varepsilon \cdot \psi_{3}+ \\
& +\left(\varepsilon \cdot \psi_{2}+\Delta \varepsilon \cdot \psi_{1}\right) \cdot \cos \left(\theta-\delta_{p}\right)=0 \\
\psi_{1}= & \frac{1}{\underline{u}} \cdot\left(x_{d} \cdot \cos ^{3} \theta+\frac{\underline{u}^{2}+x_{d^{\prime}} \alpha_{s}}{\beta_{s}} \cdot \sin ^{3} \theta\right), \\
\psi_{2}= & \frac{x_{p} \cdot \cos \delta_{p}}{\underline{u}} \cdot\left\{\frac{0,6612}{\left(1-0,57 \cdot \varepsilon_{p}\right)^{2}} \cdot\left[0,15 \cdot \varepsilon_{p}+\left(0,13 \cdot \varepsilon_{p}-0,0514 \cdot \varepsilon_{p}^{2}\right)^{\frac{1}{2}}\right]+\right. \\
& \left.+\frac{1,16}{1-0,57 \cdot \varepsilon_{p}} \cdot\left[0,15+\frac{0,13-0,1028 \cdot \varepsilon_{p}}{2 \cdot\left(0,13 \cdot \varepsilon_{p}-0,0514 \cdot \varepsilon_{p}^{2}\right)^{\frac{1}{2}}}\right]-1\right\} \\
\psi_{3}= & \frac{\sin \left(\theta-\delta_{p}\right)}{\beta_{s}} \cdot\left(\sin ^{2} \theta-\sin ^{2} \delta_{p}\right),
\end{aligned}
$$

где $\varepsilon, \Delta \varepsilon, \varepsilon_{\mathrm{p}}, \theta, \delta_{\mathrm{p}}=f\left(\alpha_{\mathrm{s}}, \beta_{\mathrm{s}}, \underline{u}\right)$ (см. выражения (9) - (13)).

Метод расчета нижних предельно допустимых значений реактивной мощности и тока возбуждения из условия осуществимости установившегося режима сводится к следующему:

1) принимают значение $\beta_{\mathrm{s}}, \underline{u}$ равными заданным и, решив уравнение (14) с учетом (15) (17), (8) - (13), находят искомое значение $\alpha_{\mathrm{s}}=\underline{\alpha}_{s}$;

2) подставив заданные значения $\beta_{\mathrm{s}}, \underline{u}$ и найденное значение $\alpha_{\mathrm{s}}$ в (8) - (13), по (12), (13) рассчитывают углы $\delta_{\mathrm{p}}, \theta$ и по (9) - (11) - ЭДС $\varepsilon, \Delta \varepsilon, \varepsilon_{\mathrm{p}}$; далее по (8) вычисляют квадрат ЭДС $\varepsilon_{0}^{2}$ и по (7) - искомое значение $\underline{I}_{2}=\underline{\dot{I}}_{2}$.

Если допустим, что

$$
x_{q}=x_{d}, \Delta \varepsilon=0,
$$

т.е. не учитывать явнополюсность ротора и изменение насыщения магнитной цепи, то уравнение (6), как нетрудно показать, примет вид

$$
x_{d}^{2} \cdot \frac{\alpha_{s}^{2}+\beta_{s}^{2}}{\underline{u}^{2}}+2 \cdot x_{d} \cdot \alpha_{s}+\underline{u}^{2}-\varepsilon_{\mathrm{H}}^{2} \cdot \underline{I}_{2}^{2}=0,
$$

а уравнение (14) - вид

$$
x_{d} \cdot \alpha_{s}+\underline{u}^{2}=0
$$

где

$$
\varepsilon_{\mathrm{H}}^{2}=x_{d}^{2}+2 \cdot x_{d} \cdot \sin \varphi_{\mathrm{H}}+1
$$

Из (18), (19)

$$
\begin{aligned}
& \alpha_{s}=\frac{\underline{u}}{x_{d}} \cdot \sqrt{\varepsilon_{\mathrm{H}}^{2} \cdot \underline{I}_{2}^{2}-x_{d}^{2} \cdot \frac{\beta_{s}^{2}}{\underline{u}^{2}}}-\frac{\underline{u}^{2}}{x_{d}}, \\
& \alpha_{s}=\underline{\alpha}_{s}=-\frac{\underline{u}^{2}}{x_{d}}
\end{aligned}
$$

Подставив в (18) вместо $\alpha_{\mathrm{s}}$ его значение из (22), имеем: 


$$
\underline{I}_{2}=\dot{I}_{2}=\frac{x_{d} \cdot \beta_{s}}{\varepsilon_{\mathrm{H}} \cdot \underline{\underline{u}}} .
$$

Найденные по формулам (21), (22) значения $\alpha_{\mathrm{s}}$ и $\underline{\alpha}_{\mathrm{s}}$ можно использовать в качестве начальных приближенных оценок корней уравнений (6) и (14).

Учитывая дифференцируемость функций $g_{2}$ и $g_{3}$ и возможность выбора по (21), (22) начального приближения, целесообразно при поиске корней нелинейных уравнений (6) и (14) использовать итерационный метод Ньютона.

Расчет установившихся режимов работы синхронного двигателя. Используя математические зависимости (5) - (17) и приближенные формулы (21) - (22), выполним расчеты установившихся режимов работы синхронного двигателя со следующими исходными данными:

$$
\cos \varphi_{\mathrm{H}}=0,9, x_{d}=0,673, x_{q}=0,448, x_{p}=0,125 .
$$

Результаты расчетов в виде графиков зависимостей $\alpha=f\left(\underline{I}_{2}\right), \alpha=f(\underline{u}), \underline{I}_{1}=f\left(\underline{I}_{2}\right), \underline{\alpha}=f(\underline{u}), \underline{\underline{I}}_{2}=f(\underline{u})$ для случая $\beta=0,72$, где

$$
\alpha=\frac{\mathrm{Q}}{\mathrm{Q}_{\mathrm{H}}}=\frac{\alpha_{S}}{\sin \varphi_{\mathrm{H}}}, \beta=\frac{P_{1}}{P_{1 \mathrm{H}}}=\frac{\beta_{S}}{\cos \varphi_{\mathrm{H}}}
$$

$P_{1 \text { н }}, Q_{\mathrm{H}}-$ номинальные значения активной (кВт) и реактивной (кВАр) мощности СД (рис. 2-6).

Для сравнения здесь же приведены значения $\alpha, \underline{I}_{1}$ полученные экспериментально.

На рис. 2-6 приняты следующие условные обозначения:

— - графики, полученные по выражениям (5) - (17);

- - - - графики, полученные по выражениям (21) - (23);

* $\quad$ - значение $\alpha$ или $\underline{I}_{1}$, полученное экспериментально.

На рис. 2-6 видно следующее:

1) использование взамен приближенных формул (21) - (23) математических зависимостей (5) - (17) существенно повышает точность расчетов установившихся режимов работы СД;

2) различия в значениях $\alpha$ и $\underline{I}_{1}$, полученных экспериментально и с использованием математических зависимостей (5) - (13), незначительны, т.е. последние достаточно точны и могут быть применены при расчетах и оптимизации установившихся режимов систем электроснабжения промышленных предприятий. 


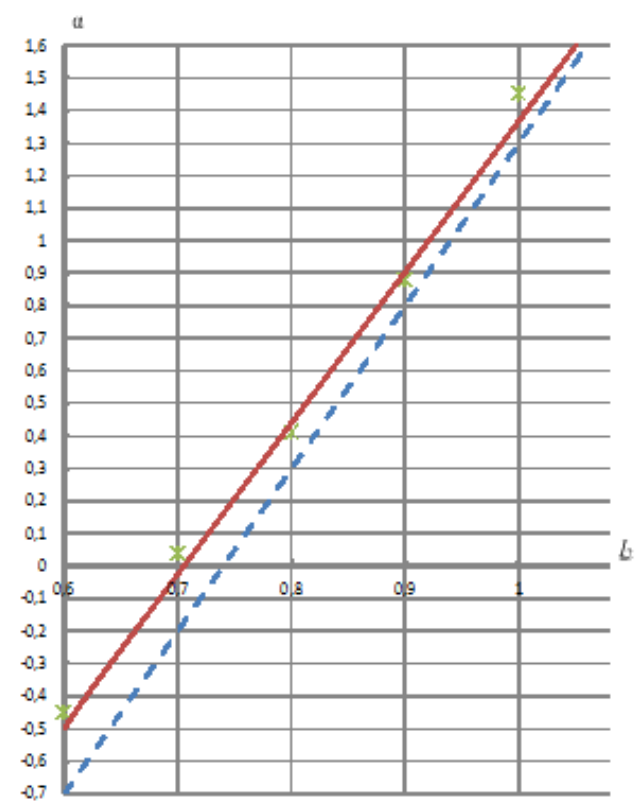

Рис. 2. График зависимости $\alpha=f\left(\underline{I}_{2}\right)$ для $\underline{u}=0,95$

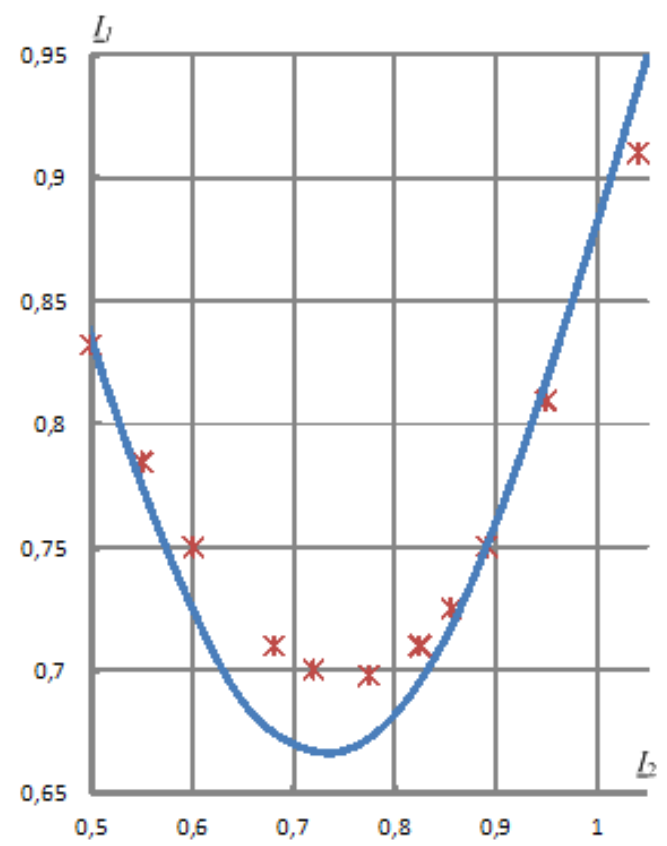

Рис. 4. Графики зависимости $\underline{I}_{1}=f\left(\underline{I}_{2}\right)$ для $\underline{u}=0,975$

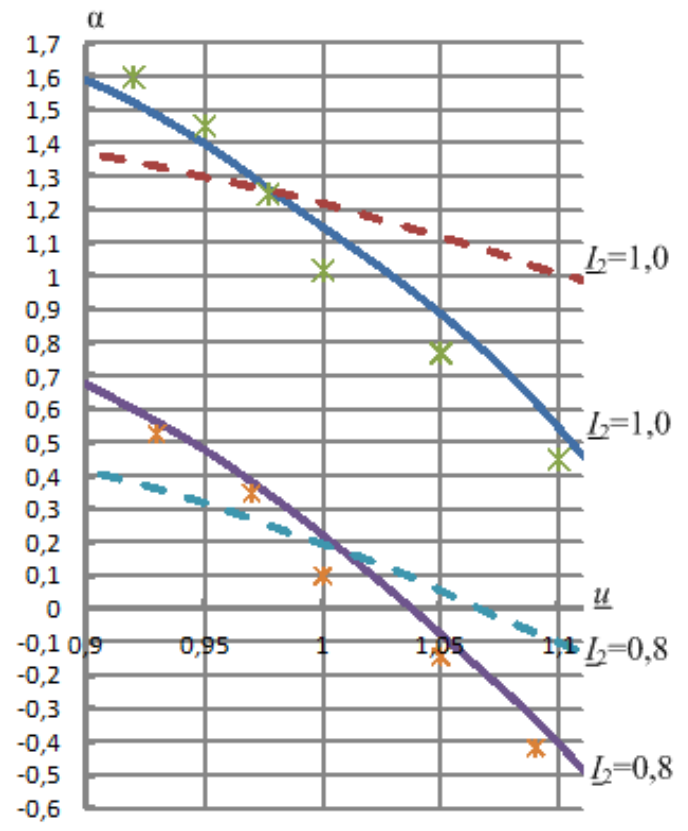

Рис. 3. Графики зависимости $\alpha=f(\underline{u})$ для $\underline{I}_{2}=1,0$ и $\underline{I}_{2}=0,8$

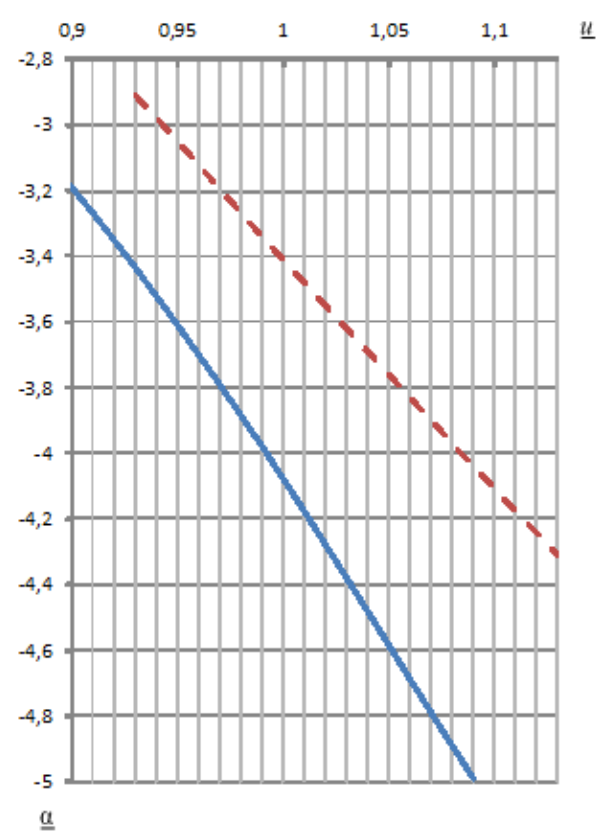

Рис. 5. Графики зависимости $\underline{\alpha}=f(\underline{u})$ 


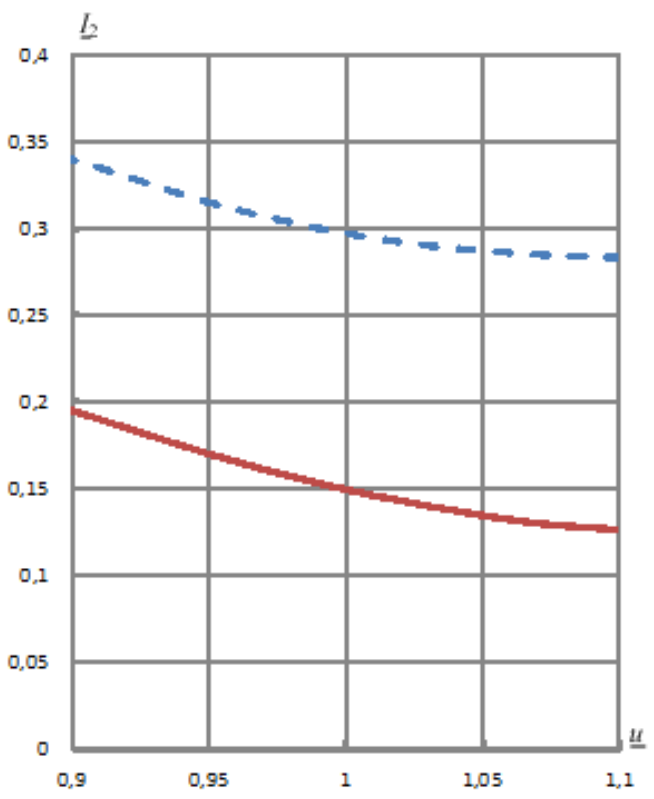

Рис. 6. Графики зависимости $\underline{\underline{I}}_{2}=f(\underline{u})$

\section{Список литературы}

[1] Архипенко В.В. // Электричество. 1993. № 2. С. 41-49.

[2] Голоднов Ю.М. // Электричество. 1999. № 2. С. 27-34. 